\title{
Aspectos clave en la vacunación contra la COVID-19 en pacientes con enfermedad inflamatoria intestinal
}

\section{Key aspects in vaccination against COVID-19 in patients with inflammatory bowel disease}

Viviana Parra-Izquierdo, ${ }^{1 *}$ (1) Sergio Andrés Remolina-Granados, ${ }^{2}$ (1) Consuelo Romero-Sánchez. ${ }^{3}$ (1)

\author{
GacCeso abierto \\ Citación: \\ Parra-Izquierdo V, Remolina-Granados SA, \\ Romero-Sánchez C. Aspectos clave en la \\ vacunación contra la COVID-19 en pacientes con \\ enfermedad inflamatoria intestinal. Rev Colomb \\ Gastroenterol. 2021:36(2):241-251. https://doi. \\ org/10.22516/25007440.785 \\ 1 Medicina interna, reumatología, \\ gastroenterología y endoscopia digestiva, Clínica \\ Palermo-Gastroadvanced IPS, Universidad \\ El Bosque, Grupo de Inmunología Celular y \\ Molecular (InMuBo). Bogotá, Colombia. \\ 2 Medicina interna, infectología, Clínica DESA \\ Clínica Nueva de Cali, Clínica Nueva Rafael \\ Uribe Uribe. Cali, Colombia. \\ 3 Universidad El Bosque, Grupo de Inmunología \\ Celular y Molecular (InMuBo). Bogotá, Colombia. \\ Hospital Militar/Universidad Militar, Grupo de \\ inmunología Clínica Aplicada. Bogotá, Colombia \\ *Correspondencia: Viviana Parra-Izquierdo. \\ vivipaz16@hotmail.com \\ Fecha recibido: $\quad 27 / 05 / 21$ \\ Fecha aceptado: 31/05/21 \\ (c) $(1) \Theta$
}

\begin{abstract}
Resumen
Actualmente, la pandemia por la enfermedad por coronavirus de 2019 (COVID-19) ha sido uno de los grandes retos para el personal de la salud y se ha convertido en un mayor desafío cuando hay pacientes con enfermedad inflamatoria intestinal (Ell); debido a la fisiopatología de la enfermedad y los tratamientos utilizados, se pueden generar interrogantes difíciles de contestar ante el corto tiempo de evidencia científica existente sobre la vacunación para coronavirus del síndrome respiratorio agudo grave de tipo 2 (SARS-CoV-2). Es claro que la vacunación es una de las intervenciones de salud pública más efectivas para lograr una inmunidad colectiva en esta pandemia; por tal motivo, se realiza una revisión enfocada en los aspectos claves frente a la vacunación para la infección por SARS-CoV-2 en los pacientes con Ell según la evidencia disponible.
\end{abstract}

\section{Palabras clave}

COVID-19, vacunas COVID-19, SARS-CoV-2, vacunación, inflamación intestinal.

\begin{abstract}
Currently, the coronavirus disease 2019 (COVID-19) pandemic has been one of the great challenges for health personnel and has become a greater challenge when we have patients with inflammatory bowel disease (IBD); because of the pathophysiology of the disease and the treatments used, it can generate questions that are difficult to answer given the short time of scientific evidence that we have on vaccination for severe acute respiratory syndrome coronavirus 2 (SARS-CoV-2). The vaccination is one of the most effective public health interventions to get immunity in this pandemic; for this reason, we made a review with the key aspects about vaccination for infection by SARS-CoV-2 in patients with IBD according to the available evidence.
\end{abstract}

Keywords

COVID-19, SARS-CoV-2, COVID-19 vaccines, vaccination, inflammatory bowel disease.

\section{INTRODUCCIÓN}

Actualmente, en la pandemia por la enfermedad por coronavirus de 2019 (COVID-19), enfermedad causada por el nuevo coronavirus del síndrome respiratorio agudo grave de tipo 2 (SARS-CoV-2), sigue habiendo una amenaza para la humanidad que requiere la realización de grandes esfuerzos en salud pública para mitigar el riesgo de infección y complicación por el virus SARS-CoV-2. Sumado a esto, se encuentra el desafío del manejo de los pacientes con enfermedad inflamatoria intestinal (EII), los cuales por la misma fisiopatología de la enfermedad y los tratamientos inmunomoduladores que se usan en ellos se vuelven un reto en el manejo médico y generan muchos interrogantes frente a su vacunación. En este momento estamos pasando por el tercer pico de esta pandemia, que nos está sorprendiendo por ser más acelerada que los dos picos anteriores; sin embargo, ante la desesperanza y 
la falta de control de los individuos, la presencia de una vacuna es causa de motivación y optimismo.

La vacunación es la forma más potente que existe para evitar las consecuencias negativas en esta pandemia. Por ahora y a corto plazo, se tiene claro que las vacunas salvarán vidas y ayudarán a evitar las formas graves de la enfermedad, lo que posibilita su control, como se ha demostrado en las miles de personas que han participado en los ensayos clínicos durante las distintas fases de desarrollo de las mismas y con los datos que se vienen obteniendo de las vacunaciones masivas.

La EII, la cual comprende la enfermedad de Crohn (EC) y la colitis ulcerativa (CU) (1), ha aumentado su prevalencia en Colombia (2) y requiere medicamentos como los corticosteroides en dosis altas (dosis de $\geq 20 \mathrm{mg}$ de prednisolona), inmunomoduladores tipo tiopurinas, metotrexato e inhibidores de la calcineurina, terapias anticitocinas incluidos los antifactor de necrosis tumoral (anti-TNF) y fármacos antiinterleucina 12/23 (anti-IL-12/23), terapias antiintegrina (vedolizumab) e inhibidores de la señalización de moléculas pequeñas (Jak/Stat-tofacitinib), que podrían dejar a estos pacientes susceptibles a la infección; por tanto, todos aquellos que tengan este tipo de inmunosupresión deben ser considerados población de alto riesgo para desarrollar COVID-19 luego del contagio (3-5). Por esta razón, todos los pacientes deben vacunarse contra la infección por SARS-CoV-2; sin embargo, hay preocupaciones sobre el impacto que puede llegar a tener la vacunación en su enfermedad.

En consecuencia, se realiza esta revisión narrativa sobre los principales interrogantes a resolver frente a este tema. Se realizó una búsqueda a través de varias bases de datos sobre la eficacia y seguridad de la vacuna en pacientes con EII en las bases de datos bibliográficas Medline (PubMed) y Scopus, con términos MeSH: ("COVID-19" O "SARSCoV-2") y ("Inflammatory bowel disease" O) y ("vacuna *" O "ARNm").

\section{¿LOS PACIENTES CON EII TIENEN MAYOR RIESGO DE INFECCIONES INDUCIDAS POR EL SARS-COV-2?}

Los coronavirus en general utilizan receptores para llegar a sus células objetivo; en este caso, se han documentado que se unen a los receptores de la enzima convertidora de angiotensina II (ECA-II) y se expresan constitutivamente por las células epiteliales del pulmón, intestino, riñón y vasos sanguíneos (6). Específicamente a nivel del tracto digestivo, se ha evidenciado que estos receptores están en mayores concentraciones en el íleon terminal y el colon (7). En el caso de los pacientes con EII en quienes hay una inflamación crónica a nivel ileal y colónica se han documentado altas concentraciones de los receptores ECA-II, que aumentan aún más su expresión durante la inflamación aguda; esto se observó en un análisis proteómico de muestras de tejido de pacientes con EII que reveló una expresión significativamente mayor de ECA-II en EC que en CU $(8,9)$.

Adicionalmente, se ha documentado en los pacientes con EII una mayor expresión de proteasas que procesan la proteína spike $(10,11)$, la cual es fundamental en el proceso de unión a los receptores ECA-II, lo que sugiere que el intestino inflamado de los pacientes con EII representa una puerta óptima para que el virus ingrese a los tejidos humanos; sin embargo, es paradójico que dentro de los reportes de poblaciones de pacientes infectados no hay hallazgos significativos que sugieran mayor gravedad en esta patología (12). Se requieren más estudios para dilucidar que otros factores adicionales pueden jugar un papel en la unión del virus a las células intestinales inflamadas.

\section{¿CUÁLES SON LAS CARACTERÍSTICAS PRINCIPALES DE LAS VACUNAS ACTUALMENTE DISPONIBLES?}

Ante la necesidad urgente de la realización de una vacuna, la ciencia ha demostrado que es posible tener una secuenciación rápida de SARS-CoV-2 y generación de varios tipos de vacunas de manera acelerada sin ningún otro precedente en la historia de esta magnitud, lo que llevó a acortar el desarrollo de estas vacunas de un tiempo que suele oscilar entre 10 y 15 años a menos de 1 año. En todo este proceso se describen varias formas para generar inmunidad contra el SARS-CoV-2. Las principales son 4 (Tabla 1 y Figura 1): virus completo (inactivado o atenuado), vector viral (replicante y no replicante), ácido nucleico (ácido desoxirribonucleico $[\mathrm{ADN}]$ y ácido ribonucleico mensajero $[\mathrm{ARNm}]$ ) y de base proteica (subunidad proteica, partícula similar al virus). Todas las vacunas intentan introducir antígenos virales al sistema inmunológico y, de esta manera, generar respuestas inmunitarias efectivas que eliminarán o impedirán que el virus ingrese a las células y cause una enfermedad, o si es infectado, generar formas leves de la enfermedad (13). Actualmente, a pesar del corto tiempo, hay información y datos acumulados que permiten entender el funcionamiento de estas. Es importante señalar que las poblaciones como niños pequeños, personas muy ancianas (> 85 años), mujeres embarazadas o en período de lactancia y que están inmunodeprimidos (ya sea debido a inmunosupresores o una enfermedad que lo inmunosuprima) fueron excluidos de estos ensayos clínicos aleatorios iniciales. Se están realizando estudios de farmacoepidemiología para evaluar los eventos adversos graves en poblaciones más específicas, incluidos estos importantes subgrupos $(14,15)$. 
Tabla 1. Características de las principales de las vacunas usadas contra el SARS-CoV-2 (16-22)

\begin{tabular}{|c|c|c|c|c|c|c|}
\hline Características & Pfizer & Sinovac & Moderna & AstraZeneca & Sputnik V & Janssen \\
\hline Tipo de vacuna & ARNm & Virus inactivado & ARNm & $\begin{array}{l}\text { Vacuna con vectores } \\
\text { virales no replicativa }\end{array}$ & $\begin{array}{l}\text { Vacuna con } \\
\text { vectores virales no } \\
\text { replicativa }\end{array}$ & $\begin{array}{l}\text { Vacuna con vectores } \\
\text { virales no replicativa }\end{array}$ \\
\hline Dosis & $\begin{array}{l}2 \text { dosis, la } \\
\text { segunda a los } \\
21 \text { días }\end{array}$ & $\begin{array}{l}2 \text { dosis, la } \\
\text { segunda a los } \\
28 \text { días }\end{array}$ & $\begin{array}{l}2 \text { dosis, la segunda a } \\
\text { los } 21 \text { días }\end{array}$ & $\begin{array}{l}2 \text { dosis, la segunda a } \\
\text { las } 4-12 \text { semanas }\end{array}$ & $\begin{array}{l}2 \text { dosis, la segunda } \\
\text { a los } 21 \text { días }\end{array}$ & 1 sola dosis \\
\hline Eficacia general & $90 \%-95 \%$ & $50 \%-65 \%$ & $94 \%$ & $70 \%-81 \%$ & $92 \%$ & $66 \%-80 \%$ \\
\hline $\begin{array}{l}\text { Eficacia contra } \\
\text { casos graves }\end{array}$ & $95 \%$ & $80 \%$ & $100 \%$ & $100 \%$ & $100 \%$ & $66 \%$ \\
\hline $\begin{array}{l}\text { Eficacia contra } \\
\text { variantes }\end{array}$ & $\begin{array}{l}95 \% \text { en las } \\
\text { variantes del } \\
\text { Reino Unido y } \\
\text { sudafricana }\end{array}$ & $\begin{array}{l}50 \% \text { contra } \\
\text { la variante } \\
\text { brasilera }\end{array}$ & $\begin{array}{l}\text { Hay efectividad } \\
\text { contra variantes, pero } \\
\text { se desconoce su } \\
\text { cuantificación (incluida } \\
\text { la variante india) }\end{array}$ & $\begin{array}{l}60 \%-77 \% \text { contra la } \\
\text { variante del Reino } \\
\text { Unido, } 21 \% \text { contra la } \\
\text { variante sudafricana }\end{array}$ & No hay información & $\begin{array}{l}\text { Hay efectividad contra } \\
\text { las variantes, pero } \\
\text { se desconoce su } \\
\text { cuantificación (incluida } \\
\text { la variante india) }\end{array}$ \\
\hline Seguras en Ell & Sí & Sí & Sí & Sí & Sí & Sí \\
\hline
\end{tabular}

\section{Virus inactivado}

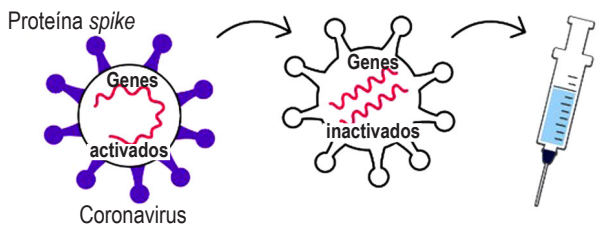

Vector viral

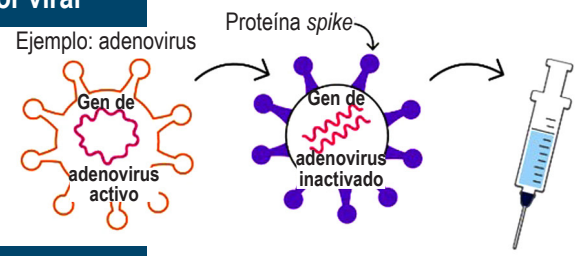

ARNm

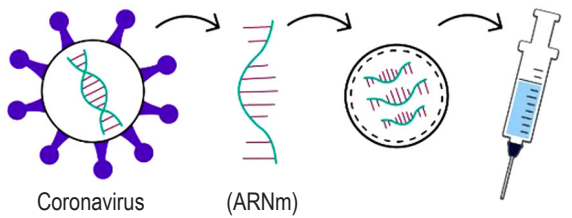

Proteínas virales
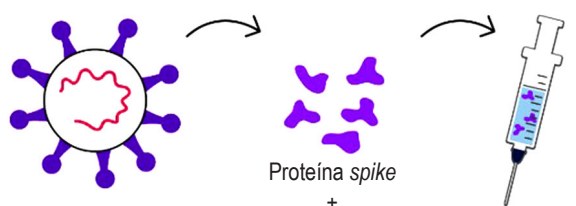

adyuvantes

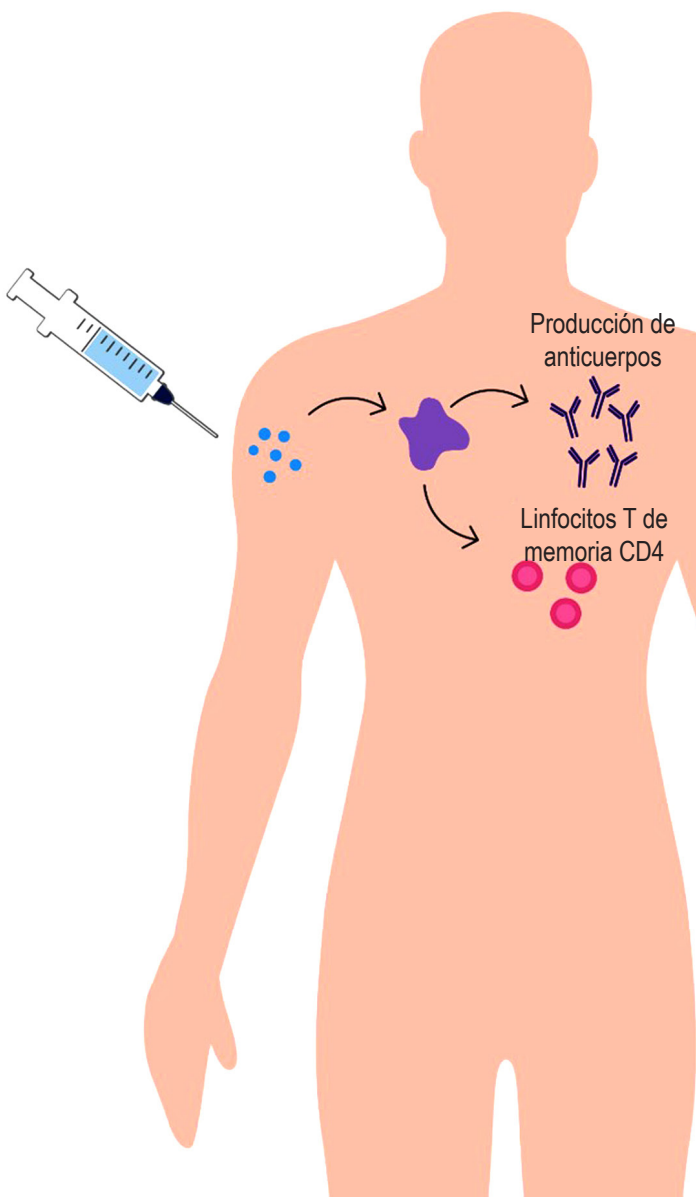

Figura 1. Mecanismos de acción de las vacunas contra SARS-CoV-2. Imagen de la Dra. Viviana Parra. 


\section{VACUNAS ANTI-COVID-19 BASADAS EN VIRUS COMPLETOS}

Estas son vacunas basadas en virus debilitados o inactivados, a través de varias tecnologías en las cuales convencionalmente trasmiten el virus a células animales o humanas que conducen a mutaciones o alteraciones a través de medidas químicas o físicas que inactivan los virus o los hacen menos virulentos. De estas vacunas de virus completos existen 2 tipos:

- Vacuna viva atenuada: esta vacuna tiene como objetivo imitar la infección natural con el fin de causar una fuerte respuesta inmunitaria por sí sola, sin necesidad de adyuvantes. El uso de todo el virus atenuado se asemeja a las infecciones naturales; por tanto, la inmunidad incluye todos los aspectos de la respuesta inmunitaria (innata y adaptativa); sin embargo, aunque son muy eficientes, estas vacunas requieren más tiempo de desarrollo, lo que retrasa el proceso. Adicionalmente, estas vacunas implican microorganismos con posibilidad de reactivarse y causar la enfermedad, por lo que en pacientes con enfermedades asociadas con el sistema inmune y en general están contraindicadas, sobre todo en aquellos que tienen terapias inmunosupresoras instauradas (23).

- Vacuna inactivada: en esta vacuna, a través de medios físicos o químicos, se inactiva el virus y con su aplicación se imita la infección natural, lo que genera una respuesta inmunológica de memoria. Estas vacunas no generan preocupación en pacientes inmunosuprimidos dado que el virus es inactivado y no tiene la capacidad de generar enfermedad $y$, por tanto, no están contraindicadas en pacientes con enfermedades asociadas con el sistema inmune que usan inmunosupresores, como la EII (23).

\section{VACUNAS CON VECTORES VIRALES}

Esta es una tecnología ya usada previamente, en la cual a través de ingeniería genética se modifica el $\mathrm{ADN}$ de un adenovirus que normalmente infecta al chimpancé o a humanos, agregando las secuencias genéticas que sintetizan la proteína $S$ o proteína spike del SARS-CoV-2 y, con su administración, producir inmunización. Como vectores para estas vacunas se utilizan virus como el sarampión o adenovirus, a los cuales se les modifican genéticamente para que no puedan causar enfermedad; estas vacunas pueden ser replicativas o no replicativas, en el caso de las que usan vectores como el sarampión o el adenovirus, respectivamente. La respuesta inmunitaria inducida por este tipo de vacunas tiende a ser fuerte, con un excelente perfil de seguridad. En el caso de individuos inmunosuprimidos, hay datos disponibles sobre vacunas basadas en vectores, cuya posibilidad de infección diseminada por parte del vec- tor es muy poco probable. Es importante destacar que la replicación del vector viral es incompetente y las vacunas a base de virus se toleran bien.

Entre las vacunas con vectores virales están Sputnik V (Gamaleya), AZD1222 (Oxford/AstraZeneca) y Covishield (Serum Institute of India) Ad5-nCoV (CanSino) y Ad26.COV2.S (Janssen), IIBR-100 (Instituto de Investigación Biológica de Israel) y De INS1-2019-nCoV-RBDOPT (Wantaj). Con algunas de estas vacunas de vectores virales se han reportado específicamente 3 casos de mielitis transversa después de la vacuna de refuerzo ChAdOx1 nCoV-19 (AZD1222); sin embargo, después del análisis por neurólogos expertos, se consideró que estos hallazgos desmielinizantes eran idiopáticos o con hallazgos preexistentes de enfermedad desmielinizante no reconocida previamente. Adicionalmente, recientemente se han descrito algunos casos de eventos trombocíticos inusuales y trombocitopenia después de la vacunación con el vector adenoviral recombinante que codifica la proteína spike del SARS-CoV-2 (ChAdOx1 nCov-19, AstraZeneca), estos 11 casos con eventos trombocíticos se presentaron entre el día 5 y el día 16 después de la vacuna y se documentó en estos pacientes la presencia de anticuerpos contra el complejo heparina-PF4, lo que configuró eventos trombóticos similares a la trombocitopenia autoinmune inducida por la heparina; sin embargo, es de aclarar que su incidencia sigue siendo muy rara y el beneficio de la vacunación siempre superará el riesgo de estos casos aislados de eventos adversos encontrados en esta vacuna $(23,24)$.

\section{VACUNA ARNM}

Está vacuna está basada en fragmentos de ARNm del virus, pero su administración no genera infección por SARS$\mathrm{CoV}-2$ en los vacunados. Estas vacunas que implementan ácidos nucleicos permiten la síntesis de la proteína $S$ de SARS-CoV-2 (en forma de ARN o ADN) por medio de un proceso en el cual se incluyen nanopartículas de lípidos para favorecer la entrada del ARN a las células huésped. Las ventajas de estos enfoques son la rapidez y facilidad del desarrollo; y dentro de las desventajas se encuentran los problemas logísticos debido a la necesidad de condiciones especiales de temperatura, dado que el ARN no es tan estable. En la línea de tiempo de las vacunas de COVID-19, las basadas en ARN son BNT162b2 (BioNTech/Pfizer), ARNm-1273 (Moderna) y CVnCoV (CureVac); las vacunas basadas en ADN son INO-4800 (Inovio) y AG0301COVID-19 (AnGes). Todos los estudios de estas vacunas evidencian en general un buen perfil de seguridad y eventos adversos comparables con el grupo de placebo y con otras vacunas virales, por lo cual se consideran seguras en los pacientes inmunosuprimidos $(16,17,23)$. 


\section{VACUNAS BASADAS EN PROTEÍNAS VIRALES}

Las vacunas basadas en subunidades proteicas consisten en fragmentos de proteínas virales o proteínas que imitan la capa externa del SARS-CoV-2. La mayoría de las vacunas que utilizan subunidades de proteínas se centra en la proteína $\mathrm{S}$, particularmente en su dominio de unión al receptor; por lo general, este tipo de vacunas requiere adyuvantes para mejorar la inmunogenicidad. Además, eventualmente, necesitan múltiples dosis para establecer una respuesta inmunitaria eficaz y duradera. Entre las vacunas que utilizan este mecanismo están RBD-Dimer (Anhui Zhifei Longcom), NVXCoV2373 (Novavax) y partículas similares al virus de origen vegetal VLP (Medicago) $(23,25)$.

\section{¿SE DEBEN VACUNAR A LOS PACIENTES CON EII?}

Actualmente existen múltiples artículos que ayudan a guiar el proceso de vacunación en pacientes con EII, entre estos se encuentra el International Organization For the Study of Inflammatory Bowel Disease (IOIBD), organización que realizó un consenso de expertos en el cual se recomienda vacunar a todos los pacientes con EII tan pronto como sea posible independientemente de la terapia inmunosupresora recibida, exceptuando las vacunas vivas atenuadas o de vectores virales replicativos que lleguen al mercado en aquellos que estén utilizando medicación inmunosupresora. Dentro de las recomendaciones, un alto porcentaje concuerda con que las vacunas disponibles no están asociadas con la aparición de EII, ni exacerbación de esta independientemente de si reciben o no terapia inmunosupresora; adicionalmente, refieren que los pacientes con EII pueden generar una respuesta inmunitaria a todas las vacunas. No se requiere ninguna modificación en el esquema de inducción ni mantenimiento en las terapias biológicas utilizadas. Así mismo, también se planteó en este consenso que la actividad de la EII no debería afectar la fecha de aplicación de la vacuna (26-29).

\section{¿EN LOS PACIENTES CON EII PUEDE MODIFICARSE LA EFECTIVIDAD DE LA VACUNACIÓN?}

La EII se maneja actualmente con terapias inmunosupresoras como los corticoesteroides, inmunomoduladores, agentes biológicos que incluyen anticuerpos monoclonales tipo inhibidores del factor de necrosis tumoral alfa (TNFa), interleucina $12 / 23$ (IL-12/23), integrinas y moléculas pequeñas como los inhibidores de la cinasa Janus (JAK). Estudios previos han evaluado la seguridad y efectividad de diferentes tipos de vacunas en pacientes con EII con especial atención al impacto de la modificación inmunológica de estas terapias sobre las respuestas serológicas, y se docu- mentó que estos pacientes sí pueden generar una respuesta inmunitaria, aunque esta puede mitigarse un poco por la terapia inmunosupresora; sin embargo, esto no se ha considerado una razón para retrasar la vacunación o suspender el tratamiento inmunosupresor (26). Se requieren registros prospectivos de pacientes con EII que reciben la vacuna contra SARS-CoV-2 para medir la amplitud y duración de la respuesta inmunitaria.

En pacientes con EII hay algunos reportes con infliximab o adalimumab en los cuales se evidencia que tienen títulos de anticuerpos disminuidos y menores tasas de seroconversión en comparación con controles en respuesta a la vacunación con virus inactivados de la influenza (30), neumococo (31) y el virus de la hepatitis B (32); la respuesta puede verse atenuada aún más por las tiopurinas y metotrexato administrados solos o en combinación con terapia anti-TNF (33). Los pacientes con otras enfermedades inmunomediadas como la artritis reumatoide, quienes usaban el inhibidor de JAK tipo tofacitinib, tuvieron una respuesta normal de anticuerpos contra la vacuna de la influenza y una disminución en la respuesta a la vacuna neumocócica (34). El bloqueo de IL-12/23 con ustekinumab no parece alterar la respuesta a la vacuna de la influenza (35) ni neumococo (36), y de manera similar, los pacientes con EII tratados con vedolizumab no tienen respuestas inmunitarias alteradas a la vacuna contra la influenza (37); sin embargo, el vedolizumab sí tiene un impacto con disminución en la eficacia de la vacuna contra el cólera (38), la cual es de administración oral y puede reducir la eficacia de vacunas administradas por las mucosas, lo cual puede ser relevante para algunas vacunas orales contra el SARSCoV-2 que actualmente están en desarrollo.

Por todo lo anterior, en los consensos y artículos que revisan la vacunación en pacientes con EII se recomienda que la vacunación no debe posponerse si el paciente está recibiendo ácido 5-aminosalicílico (5-ASA) oral o tópico, corticoesteroides sistémicos, tiopurinas, metotrexato, terapia biológica o moléculas pequeñas como los inhibidores de JAK, así mismo también se sugiere que no se retrase la vacunación si estos pacientes están en algún ensayo clínico de algún medicamento para la EII (26-28).

\section{¿QUÉ OTROS FACTORES PUEDEN MODIFICAR LA EFECTIVIDAD DE LA VACUNA?}

Es importante tener en cuenta que, por una parte, adicional a los medicamentos inmunosupresores, existen otros factores que pueden modificar la respuesta a las vacunas en los pacientes con EII, como la edad en el caso de los pacientes adultos mayores y en algunos estudios se sugiere que el sexo masculino puede asociarse con más bajas tasas de seroconversión posvacunación (39). Por otra parte, las 
comorbilidades como la obesidad, desnutrición o daño a órganos blanco específicos pueden alterar la respuesta a la vacuna por alteración en el funcionamiento de las células $\mathrm{T}$ y reducción de los niveles de interferón gamma y granzima B (40). Igualmente, también se sugiere que las infecciones preexistentes o infecciones previas por otros coronavirus pueden generar lo que se llama impronta inmunológica (41), que afecta la efectividad de las vacunas. También se ha observado en algunas enfermedades autoinmunes que, cuando estas han sido de larga duración o presentan actividad de la enfermedad, estos factores pueden disminuir las tasas de seroconversión de las vacunas (42).

\section{¿SON SEGURAS LAS VACUNAS PARA COVID-19 EN PACIENTES CON EII?}

En los ensayos clínicos iniciales de las vacunas que se han desarrollado y aprobado no se incluyeron pacientes con patologías como la EII; sin embargo, en general las vacunas no vivas se consideran seguras en estos pacientes y actualmente todas las vacunas que se están aplicando en nuestro país son vacunas no vivas; es decir, inactivadas. Se requieren estudios de poblaciones de pacientes con EII en las cuales se analicen eventos adversos específicos. La IOIBD en su consenso recomienda que las vacunas en EII son seguras, no están relacionadas con aparición de EII ni con exacerbación de la enfermedad independiente de si está o no recibiendo terapia inmunosupresora y siempre es de aclarar que las vacunas con virus vivos siempre estarán contraindicadas en aquellos pacientes con EII que reciban terapia inmunosupresora (26). La Sociedad Británica de Gastroenterología ha publicado recientemente recomendaciones claves que apoyan firmemente la vacunación contra el SARS-CoV-2 en pacientes con EII y enfatizan en que la principal preocupación en pacientes tratados con agentes biológicos o moléculas pequeñas es el riesgo teórico de respuestas subóptimas a la vacuna en lugar de los efectos adversos de la vacuna (28). Sin embargo, el riesgo de morbilidad y mortalidad asociadas con complicaciones de COVID-19 supera con creces el riesgo que pudiera tener la vacunación.

Con respecto a las reacciones que pueden producir las vacunas, es importante distinguir siempre entre un evento adverso y un efecto secundario. El evento adverso es un problema de salud después de una vacuna que podría o no ser causado por la vacuna, mientras que los efectos secundarios son problemas de salud causados directamente por la vacuna. Los eventos adversos pueden requerir tratamiento, mientras que la mayoría de los efectos secundarios se resuelve espontáneamente con el tiempo. En todos los ensayos clínicos se ha documentado un buen perfil de seguridad de las vacunas actuales para la COVID-19; los efectos secun- darios más frecuentemente descritos han sido reacciones locales en el sitio de la aplicación como dolor, prurito, eritema, inflamación e induración. Así mismo, se han presentado algunas reacciones sistémicas tales como fatiga, cefalea, mialgias, artralgias, malestar general y pérdida del apetito (16-22). La mayoría de los efectos secundarios han sido leves a moderados y transitorios, por lo cual es muy importante siempre dar signos de alarma a todos los pacientes que serán llevados a vacunación y monitorizar al menos por 30 minutos a los pacientes inmediatamente después de la vacuna para descartar efectos secundarios inmediatos. En conclusión, las observaciones hasta el momento indican que, en general, las vacunas contra COVID-19 son seguras. En la actualidad, no hay evidencia disponible que contraindique la administración de las vacunas en pacientes con enfermedades mediadas por el sistema inmune. Sin embargo, se deben instaurar programas de farmacovigilancia en los que todos los pacientes con EII que reciban la vacuna puedan participar.

\section{¿SE PUEDEN VACUNAR PACIENTES EMBARAZADAS CON EII?}

Como se ha mencionado anteriormente, los ensayos clínicos de las vacunas disponibles han excluido a población gestante y que estén lactando, por lo cual siempre se genera un debate frente a este tema; sin embargo, en el consenso de la IOIBD las vacunas contra el SARS-CoV-2 se consideran seguras en embarazadas sin EII y no hay ningún otro factor que debiera excluir a las pacientes embarazadas con EII para tener el beneficio de la vacuna, por lo cual se consideran seguras en esta población especial (26). Es importante tener en cuenta que las mujeres en gestación tienen una mayor probabilidad de cuadro grave, con mayor morbimortalidad, que afecta paralelamente el binomio madrefeto; motivo por el cual el riesgo/beneficio de la vacunación implica la posibilidad de que esta población acceda a esta medida de prevención; lo anteriormente mencionado se vio reflejado en un estudio en el que se documentó que las mujeres embarazadas tenían una probabilidad significativamente mayor que las mujeres no embarazadas de ser ingresadas en una unidad de cuidados intensivos (UCI), recibir ventilación y recibir oxigenación por membrana extracorpórea (OMEC) (43).

\section{¿LA VACUNACIÓN PARA COVID-19 PUEDE PRODUCIR FENÓMENOS AUTOINMUNES?}

Teóricamente, la autoinmunidad puede ser potencialmente desencadenada por vacunas (44), mecanismos inmunológicos como la presentación de antígenos, producción de 
citocinas, redes antiidiotípicas, activación y propagación de epítopos y activación policlonal de las células B que están involucradas tanto en la respuesta inmunitaria antiinfecciosa como en la autorreactividad $(45,46)$. Se ha documentado presencia de anticuerpos antinucleares y respuesta celular de tipo autoinmune durante y después de las infecciones virales; sin embargo, esta positividad autorreactiva suele ser transitoria y, por lo general, no es seguida de síntomas o consecuencias clínicas $(47,48)$. La autorreactividad no es igual a la autoinmunidad debido a que existen varios y suficientes mecanismos de control que regulan las respuestas del sistema inmunológico; además, existe una fuerte evidencia de que el desarrollo de las enfermedades autoinmunes depende de muchos otros factores $y$, adicionalmente, ocurren en ausencia del estímulo generado por la vacunación (49). La posible asociación positiva entre vacunas y la autoinmunidad se basó principalmente en casos anecdóticos, informes de casos y estudios observacionales no controlados. Por ejemplo, Campylobacter se vinculó con el síndrome de Guillain-Barré (GBS), virus de la influenza con esclerosis múltiple, virus Coxsackie con diabetes tipo 1 y parvovirus humano B19 con artritis reumatoide, lo que evidencia que por sí misma la infección natural puede provocar trastornos autoinmunitarios (49-54).

Hasta la fecha, los registros de los ensayos clínicos dan resultados tranquilizadores frente a este tema de autoinmunidad inducia por la vacuna. Se han registrado algunos eventos considerados raros, como los que se informan por la vacuna de AstraZeneca, por los que se suspendió temporalmente el estudio de Oxford debido a la presencia de casos de mielitis transversa (55). Como se explicó anteriormente, se realizaron las evaluaciones por neurólogos expertos y se consideraron casos idiosincráticos y algunos causados en pacientes con estigmas de enfermedades desmielinizantes silentes. Recientemente, también se publicó la descripción de algunos casos raros de fenómenos trombóticos asociados con anticuerpos contra proteínas plaquetarias que asemejaban los eventos trombóticos autoinmunes asociados con trombocitopenia inducida por heparina, que los autores del artículo sugirieron denominar como trombocitopenia trombótica inmunitaria inducida por vacunas (VITT), para evitar confusión con la trombocitopenia inducida por heparina (56); sin embargo, en el caso de estas vacunas de vectores virales sigue primando el beneficio de la vacuna frente a los riesgos muy bajos de efectos secundarios, como la generación de fenómenos autoinmunes.

Con respecto a otras tecnologías de vacunas, las vacunas con base en ARNm pueden comportarse como patrones moleculares asociados con daño y activar los receptores toll tipo TLR7 y TLR8, lo que da como resultado la producción de interferón tipo I (57). Según algunos estudios de vacunas con este tipo de mecanismo de acción, también pueden aumentar la producción de citocinas y quimiocinas después de la aplicación intradérmica, producir estimulación de la maduración de las células dendríticas, provocar respuestas robustas de células $\mathrm{T}$ y $\mathrm{B}, \mathrm{y}$ activar linfocitos autorreactivos transitorios. Teóricamente, este proceso puede reactivar enfermedades autoinmunes, pero el ARNm exhibe una función inhibidora sobre la expresión de antígenos, que puede suprimir la respuesta inmunitaria $(58,59)$. Por tanto, a pesar de estos reportes (que, como lo hemos expresado, son muy raros), se enfatiza en que es poco probable que las vacunas para la COVID-19 tengan una relación directa y significativa con la generación de procesos autoinmunes $\mathrm{u}$ otros trastornos inflamatorios en los participantes de los ensayos clínicos hasta ahora realizados comparados con placebo.

\section{¿SE DEBEN PRIORIZAR A LOS PACIENTES CON EII PARA LA VACUNACIÓN CONTRA LA COVID-19?}

No se conoce claramente si los pacientes con EII tienen un riesgo mayor de contraer la infección por SARS-CoV-2 que las personas sin EII con niveles similares de exposición viral, pero es poco probable que el riesgo sea menor, como se ha explicado anteriormente. La evidencia hasta la fecha sugiere que los pacientes con EII que tienen COVID-19, incluidos aquellos en manejo con terapia biológica a largo plazo e inmunomoduladores no esteroideos, pueden no tener un mayor riesgo de resultados graves como hospitalización y muerte (12) en comparación con pacientes con COVID-19 sin EII. Sin embargo, el uso reciente de corticosteroides a dosis altas puede estar asociado con un mayor riesgo de resultados graves de COVID-19. Los factores de riesgo de resultados graves de COVID-19 en pacientes con EII parece ser similar a las ampliamente reconocidas en pacientes con COVID-19 sin EII. Estos factores de riesgo incluyen edad avanzada y múltiples comorbilidades (12). En algunas revisiones se sugiere priorizar a los pacientes con EII con otros factores de riesgo adicionales, como el uso de corticoesteroides $\geq 20 \mathrm{mg} /$ día equivalente de prednisolona y con actividad de la enfermedad de moderada a grave, y también si estos pacientes con EII tienen un riesgo ocupacional como ser trabajadores de la salud, profesores $\mathrm{u}$ otros trabajadores esenciales.

En el consenso de la IOIBD refieren que las personas que no son trabajadores de atención médica y no tienen factores de riesgo de complicaciones de COVID-19, pero tienen EII deben vacunarse en el mismo nivel de priorización que aquellos que no son de atención médica y no tienen factores de riesgo para el SARS-COV-2. A su vez, plantean que los individuos con EII que están en trata- 
miento inmunosupresor, pero no tienen riesgo de complicaciones de COVID-19 deben vacunarse en el mismo nivel de prioridad que aquellos que están inmunosuprimidos por otras causas, de acuerdo con las recomendaciones regionales (26).

En Colombia, la Asociación Colombiana de Infectología indica que los pacientes con enfermedad autoinmune, incluidos aquellos con EII que están en tratamiento inmunomodulador o biológico, requieren priorización de vacuna al mismo nivel que las personas con factores de riesgo para mal pronóstico. De igual forma, se aclara que no hay contraindicación de la vacunación en esta población y en la evaluación riesgo-beneficio se debe acceder a esta estrategia de prevención (60). La Asociación Colombiana de Gastroenterología también emitió un comunicado al Ministerio de Salud y Protección Social en la que se solicitó la priorización de esta población con EII y factores de riesgo para enfermedad grave por COVID-19.

En múltiples revisiones y consensos (26-29, 61-63) en los que abordan este tema en pacientes con enfermedades autoinmunes, las cuales son de bases muy heterogéneas en sus manifestaciones, gravedad e intensidad del manejo inmunosupresor, se plantean que si los pacientes sufren infección por SARS-CoV-2, podrían tener mayor riesgo de hospitalización y peores resultados que la población general, por lo que se sugiere que este grupo de pacientes debería ser priorizado para ser vacunado antes que la población no priorizada de similar edad y sexo. En conclusión, los pacientes con EII deben vacunarse de acuerdo con su riesgo general de exposición y riesgo de complicaciones por SARS-CoV-2. Estos riesgos continúan siendo investigados en los registros de vigilancia epidemiológica, como los de SECURE IBD y otros basados en poblaciones de estudios.

\section{CONCLUSIÓN}

La pandemia de SARS-CoV-2 que estamos experimentando actualmente ha tenido un impacto sin precedentes en nuestra salud pública y lastimosamente no existe un tratamiento ampliamente eficaz en el momento disponible; por tal motivo, se requiere la adquisición de una inmunidad colectiva por medio de la vacunación para poder romper con esa cadena de transmisión. Los pacientes con EII son una población especial, dado que es una enfermedad inmunomediada que requiere el uso de terapia inmunosupresora para controlarla; por tanto, la aprobación de la vacuna contra el SARS-CoV-2 crea una urgencia para desarrollar recomendaciones para pacientes con EII.

Con las revisiones y consensos mundiales publicados actualmente con respecto a la vacunación contra SARS$\mathrm{CoV}-2$ en pacientes con EII se concluye que deben ser vacunados con cualquiera de las vacunas no vivas que actualmente están en el mercado y el mejor tiempo para hacerlo es en la primera oportunidad que tenga el paciente según sus factores de riesgo, estas vacunas son seguras y no se debe retrasar su aplicación independientemente del tratamiento instaurado ni de la actividad de la enfermedad; sin embargo, se enfatiza en que el gastroenterólogo debe realizar un asesoramiento a los pacientes con EII sobre su eficacia y la posible disminución de la seroconversión, especialmente con el uso de corticoides sistémicos. Se necesitan estudios para definir si el título de anticuerpos debe controlarse después la vacunación en esta población de pacientes y, de ser así, con qué frecuencia y durante cuánto tiempo. Finalmente, se debe realizar un seguimiento de los pacientes con EII que reciben vacunas contra el SARS-CoV-2 para asegurar la mejor estrategia preventiva y $\mathrm{NO}$ olvidar que la principal prioridad es la administración de la vacuna lo antes posible.

\section{REFERENCIAS}

1. Abraham C, Cho JH. Inflammatory bowel disease. N Engl J Med. 2009;361(21):2066-78. https://doi.org/10.1056/NEJMra0804647

2. Fernández-Ávila DG, Bernal-Macías S, Parra-Izquierdo V, Rincón-Riaño DN, Gutiérrez JM, Rosselli D. Prevalencia en Colombia de la enfermedad inflamatoria intestinal y el compromiso articular asociado, según información del Sistema Integral de Información de la Protección Social. Rev Colomb Reumatol. 2020;27(1):3-8. https://doi.org/10.1016/j.rcreu.2019.10.006

3. Saavedra Trujillo CH. Vacunación. Infectio. $2021 ; 25(4$ suppl 1):250-85. https://doi.org/10.22354/in.v25i4.978

4. Kirchgesner J, Lemaitre M, Carrat F, Zureik M, Carbonnel F, Dray-Spira R. Risk of Serious and Opportunistic Infections
Associated With Treatment of Inflammatory Bowel Diseases. Gastroenterology. 2018;155(2):337-346.e10. https://doi.org/10.1053/j.gastro.2018.04.012

5. Lichtenstein GR, Feagan BG, Cohen RD, Salzberg BA, Diamond RH, Price $\mathrm{S}$, et al. Serious infection and mortality in patients with Crohn's disease: more than 5 years of follow-up in the TREAT ${ }^{\mathrm{Ts}}$ registry. Am J Gastroenterol. 2012;107(9):1409-22. https://doi.org/10.1038/ajg.2012.218

6. Hoffmann M, Kleine-Weber H, Schroeder S, Krüger N, Herrler T, Erichsen S, et al. SARS-CoV-2 Cell Entry Depends on ACE2 and TMPRSS2 and Is Blocked by a Clinically Proven Protease Inhibitor. Cell. 
2020;181(2):271-280.e8.

https://doi.org/10.1016/j.cell.2020.02.052

7. Harmer D, Gilbert M, Borman R, Clark KL. Quantitative mRNA expression profiling of ACE 2, a novel homologue of angiotensin converting enzyme. FEBS Lett. 2002;532(12):107-10. https://doi.org/10.1016/s0014-5793(02)03640-2

8. Garg M, Royce SG, Tikellis C, Shallue C, Batu D, Velkoska $\mathrm{E}$, et al. Imbalance of the renin-angiotensin system may contribute to inflammation and fibrosis in IBD: a novel therapeutic target? Gut. 2020;69(5):841-851. https://doi.org/10.1136/gutjnl-2019-318512

9. Ning L, Shan G, Sun Z, Zhang F, Xu C, Lou X, et al. Quantitative Proteomic Analysis Reveals the Deregulation of Nicotinamide Adenine Dinucleotide Metabolism and CD38 in Inflammatory Bowel Disease. Biomed Res Int. 2019;2019:3950628.

https://doi.org/10.1155/2019/3950628

10. Ibrahim IM, Abdelmalek DH, Elshahat ME, Elfiky AA. COVID-19 spike-host cell receptor GRP78 binding site prediction. J Infect. 2020;80(5): 554-562. https://doi.org/10.21203/rs.2.24599/v1

11. Zhang H, Kang Z, Gong H, Xu D, Wang J, Li Z, et al. The digestive system is a potential route of 2019nCov infection: a bioinformatics analysis based on single-cell transcriptomes. bioRxiv. 31 de enero de 2020;2020.01.30.927806.

https://doi.org/10.1101/2020.01.30.927806

12. Kappelman M, Brenner E, Colombel JF, Ungaro R, Agrawal $\mathrm{M}$. Coronavirus and IBD Reporting Database [Internet]. Secure-IBD Database [citado el 1 de abril de 2020]. Disponible en: https://covidibd.org/

13. Pollard AJ, Bijker EM. A guide to vaccinology: from basic principles to new developments. Nat Rev Immunol. 2021;21(2):83-100. https://doi.org/10.1038/s41577-020-00479-7

14. Draft landscape and tracker of COVID-19 candidate vaccines [Internet]. World Health Organization [citado el 20 de mayo de 2021]. Disponible en: https://www.who.int/ publications $/ \mathrm{m} /$ item/draft-landscape-of-covid-19-candidate-vaccines

15. Thanh Le T, Andreadakis Z, Kumar A, Gómez Román R, Tollefsen S, Saville M, et al. The COVID-19 vaccine development landscape. Nat Rev Drug Discov. 2020;19(5):305-306. https://doi.org/10.1038/d41573-020-00073-5

16. Polack FP, Thomas SJ, Kitchin N, Absalon J, Gurtman A, Lockhart S, Perez JL, et al. Safety and Efficacy of the BNT162b2 mRNA Covid-19 Vaccine. N Engl J Med. 2020;383(27):2603-2615. https://doi.org/10.1056/NEJMoa2034577

17. Baden LR, El Sahly HM, Essink B, Kotloff K, Frey S, Novak $\mathrm{R}$, et al. Efficacy and Safety of the mRNA-1273 SARSCoV-2 Vaccine. N Engl J Med. 2021;384(5):403-416. https://doi.org/10.1056/NEJMoa2035389

18. SadoffJ, Gray G, Vandebosch A, Cárdenas V, Shukarev G, Grinsztejn B, et al. Safety and Efficacy of Single-Dose Ad26.
COV2.S Vaccine against Covid-19. N Engl J Med. 2021. https://doi.org/10.1056/NEJMoa2101544

19. Logunov DY, Dolzhikova IV, Shcheblyakov DV, Tukhvatulin AI, Zubkova OV, Dzharullaeva AS, et al. Safety and efficacy of an rAd26 and rAd5 vector-based heterologous prime-boost COVID-19 vaccine: an interim analysis of a randomised controlled phase 3 trial in Russia. Lancet. 2021;397(10275):671-681. https://doi.org/10.1016/S0140-6736(21)00234-8

20. Voysey M, Clemens SAC, Madhi SA, Weckx LY, Folegatti PM, Aley PK, et al. Safety and efficacy of the ChAdOx1 nCoV-19 vaccine (AZD1222) against SARS-CoV-2: an interim analysis of four randomised controlled trials in Brazil, South Africa, and the UK. Lancet. 2021;397(10269):99-111. https://doi.org/10.1016/S0140-6736(20)32661-1

21. Zhang Y, Zeng G, Pan H, Li C, Hu Y, Chu K, et al. Safety, tolerability, and immunogenicity of an inactivated SARSCoV-2 vaccine in healthy adults aged $18-59$ years: a randomised, double-blind, placebo-controlled, phase $1 / 2$ clinical trial. Lancet Infect Dis. 2021;21(2):181-192. https://doi.org/10.1016/S1473-3099(20)30843-4

22. Wu Z, Hu Y, Xu M, Chen Z, Yang W, Jiang Z, et al. Safety, tolerability, and immunogenicity of an inactivated SARS-CoV-2 vaccine (CoronaVac) in healthy adults aged 60 years and older: a randomised, double-blind, placebo-controlled, phase $1 / 2$ clinical trial. Lancet Infect Dis. 2021;21(6):803-812. https://doi.org/10.1016/S1473-3099(20)30987-7

23. Principles of Vaccination. En: Epidemiology and Prevention of Vaccine-Preventable Diseases [Internet]. 13. ${ }^{\mathrm{a}}$ edición. CDC; 2015 [actualizado el 29 de junio de 2020; citado el 20 de mayo de 2021]. Disponible en: https:// www.cdc.gov/vaccines/pubs/pinkbook/prinvac.html

24. Voysey M, Costa Clemens SA, Madhi SA, Weckx LY, Folegatti PM, Aley PK, et al. Safety and efficacy of the ChAdOx1 nCoV-19 vaccine (AZD1222) against SARS-CoV-2: an interim analysis of four randomised controlled trials in Brazil, South Africa, and the UK. 2021;397(10269):99-11. https://doi.org/10.1016/ S0140-6736(20)32661-1

25. Vartak A, Sucheck SJ. Recent Advances in Subunit Vaccine Carriers. Vaccines (Basel). 2016;4(2):12. https://doi.org/10.3390/vaccines4020012

26. Rubin DT, Abreu MT, Rai V, Siegel CA; International Organization for the Study of Inflammatory Bowel Disease. Management of Patients With Crohn's Disease and Ulcerative Colitis During the Coronavirus Disease-2019 Pandemic: Results of an International Meeting. Gastroenterology. 2020;159(1):6-13.e6. https://doi.org/10.1053/j.gastro.2020.04.002

27. Siegel CA, Melmed GY, McGovern DP, Rai V, Krammer F, Rubin DT, et al. SARS-CoV-2 vaccination for patients with inflammatory bowel diseases: recommendations from an international consensus meeting. Gut. 2021;70(4):635-640. https://doi.org/10.1136/gutjnl-2020-324000 
28. Alexander JL, Moran GW, Gaya DR, Raine T, Hart A, Kennedy NA, et al. SARS-CoV-2 vaccination for patients with inflammatory bowel disease: a British Society of Gastroenterology Inflammatory Bowel Disease section and IBD Clinical Research Group position statement. Lancet Gastroenterol Hepatol. 2021;6(3):218-224. https://doi.org/10.1016/S2468-1253(21)00024-8

29. D’Amico F, Rabaud C, Peyrin-Biroulet L, Danese S. SARSCoV-2 vaccination in IBD: more pros than cons. Nat Rev Gastroenterol Hepatol. 2021;18(4):211-213. https://doi.org/10.1038/s41575-021-00420-w

30. deBruyn J, Fonseca K, Ghosh S, Panaccione R, Gasia MF, Ueno A, et al. Immunogenicity of Influenza Vaccine for Patients with Inflammatory Bowel Disease on Maintenance Infliximab Therapy: A Randomized Trial. Inflamm Bowel Dis. 2016;22(3):638-47. https://doi.org/10.1097/MIB.0000000000000615

31. Melmed GY, Agarwal N, Frenck RW, Ippoliti AF, Ibanez P, Papadakis KA, et al. Immunosuppression impairs response to pneumococcal polysaccharide vaccination in patients with inflammatory bowel disease. Am J Gastroenterol. 2010;105(1):148-54. https://doi.org/10.1038/ajg.2009.523

32. Pratt PKJr, David N, Weber HC, Little FF, Kourkoumpetis T, Patts GJ, et al. Antibody Response to Hepatitis B Virus Vaccine is Impaired in Patients With Inflammatory Bowel Disease on Infliximab Therapy. Inflamm Bowel Dis. 2018;24(2):380-386. https://doi.org/10.1093/ibd/izx001

33. Agarwal N, Ollington K, Kaneshiro M, Frenck R, Melmed GY. Are immunosuppressive medications associated with decreased responses to routine immunizations? A systematic review. Vaccine. 2012;30(8):1413-24. https://doi.org/10.1016/j.vaccine.2011.11.109

34. Winthrop KL, Silverfield J, Racewicz A, Neal J, Lee EB, Hrycaj $P$, et al. The effect of tofacitinib on pneumococcal and influenza vaccine responses in rheumatoid arthritis. Ann Rheum Dis. 2016;75(4):687-95. https://doi.org/10.1136/annrheumdis-2014-207191

35. Doornekamp L, Goetgebuer RL, Schmitz KS, Goeijenbier M, van der Woude CJ, Fouchier R, et al. High Immunogenicity to Influenza Vaccination in Crohn's Disease Patients Treated with Ustekinumab. Vaccines (Basel). 2020;8(3):455. https://doi.org/10.3390/vaccines 8030455

36. Brodmerkel C, Wadman E, Langley RG, Papp KA, Bourcier $\mathrm{M}$, Poulin $\mathrm{Y}$, et al. Immune response to pneumococcus and tetanus toxoid in patients with moderate-to-severe psoriasis following long-term ustekinumab use. J Drugs Dermatol. 2013;12(10):1122-9.

37. Caldera F, Hillman L, Saha S, Wald A, Grimes I, Zhang Y, et al. Immunogenicity of High Dose Influenza Vaccine for Patients with Inflammatory Bowel Disease on Anti-TNF Monotherapy: A Randomized Clinical Trial. Inflamm Bowel Dis. 2020;26(4):593-602. https://doi.org/10.1093/ibd/izz164
38. Wyant T, Leach T, Sankoh S, Wang Y, Paolino J, Pasetti MF, et al. Vedolizumab affects antibody responses to immunisation selectively in the gastrointestinal tract: randomised controlled trial results. Gut. 2015;64(1):77-83. https://doi.org/10.1136/gutjnl-2014-307127

39. Flanagan KL, Fink AL, Plebanski M, Klein SL. Sex and Gender Differences in the Outcomes of Vaccination over the Life Course. Annu Rev Cell Dev Biol. 2017;33:577-599. https://doi.org/10.1146/annurev-cellbio-100616-060718

40. Sheridan PA, Paich HA, Handy J, Karlsson EA, Hudgens $M G$, Sammon AB, et al. Obesity is associated with impaired immune response to influenza vaccination in humans. Int J Obes (Lond). 2012;36(8):1072-7. https://doi.org/10.1038/ijo.2011.208

41. Dhakal S, Klein SL. Host Factors Impact Vaccine Efficacy: Implications for Seasonal and Universal Influenza Vaccine Programs. J Virol. 2019;93(21):e00797-19. https://doi.org/10.1128/JVI.00797-19

42. Campos LM, Silva CA, Aikawa NE, Jesus AA, Moraes JC, Miraglia J, et al. High disease activity: an independent factor for reduced immunogenicity of the pandemic influenza a vaccine in patients with juvenile systemic lupus erythematosus. Arthritis Care Res (Hoboken). 2013;65(7):1121-7. https://doi.org/10.1002/acr.21948

43. Ellington S, Strid P, Tong VT, Woodworth K, Galang $\mathrm{RR}$, Zambrano LD, et al. Characteristics of Women of Reproductive Age with Laboratory-Confirmed SARSCoV-2 Infection by Pregnancy Status - United States, January 22-June 7, 2020. MMWR Morb Mortal Wkly Rep. 2020;69(25):769-775. https://doi.org/10.15585/mmwr.mm6925a 1

44. Vera-Lastra O, Medina G, Cruz-Dominguez Mdel P, Jara LJ, Shoenfeld Y. Autoimmune/inflammatory syndrome induced by adjuvants (Shoenfeld's syndrome): clinical and immunological spectrum. Expert Rev Clin Immunol. 2013;9(4):361-73. https://doi.org/10.1586/eci.13.2

45. Münz C, Lünemann JD, Getts MT, Miller SD. Antiviral immune responses: triggers of or triggered by autoimmunity? Nat Rev Immunol. 2009;9(4):246-58. https://doi.org/10.1038/nri2527

46. Agmon-Levin N, Paz Z, Israeli E, Shoenfeld Y. Vaccines and autoimmunity. Nat Rev Rheumatol. 2009;5(11):648-52. https://doi.org/10.1038/nrrheum.2009.196

47. Schattner A. Consequence or coincidence? The occurrence, pathogenesis and significance of autoimmune manifestations after viral vaccines. Vaccine. 2005;23(30):3876-86. https://doi.org/10.1016/j.vaccine.2005.03.005

48. Salemi S, D’Amelio R. Could autoimmunity be induced by vaccination? Int Rev Immunol. 2010;29(3):247-69. https://doi.org/10.3109/08830181003746304

49. Vadalà $M$, Poddighe $\mathrm{D}$, Laurino $\mathrm{C}$, Palmieri B. Vaccination and autoimmune diseases: is prevention of adverse health effects on the horizon? EPMA J. 2017;8(3):295-311. https://doi.org/10.1007/s13167-017-0101-y 
50. De Martino M, Chiappini E, Galli L. Vaccines and autoimmunity. Int J Immunopathol Pharmacol. 2013;26(2):283-90. https://doi.org/10.1177/039463201302600201

51. Rodríguez Y, Rojas M, Pacheco Y, Acosta-Ampudia Y, Ramírez-Santana C, Monsalve DM, et al. Guillain-Barré syndrome, transverse myelitis and infectious diseases. Cell Mol Immunol. 2018;15(6):547-562. https://doi.org/10.1038/cmi.2017.142

52. Markovic-Plese S, Hemmer B, Zhao Y, Simon R, Pinilla C, Martin R. High level of cross-reactivity in influenza virus hemagglutinin-specific CD4+ T-cell response: implications for the initiation of autoimmune response in multiple sclerosis. J Neuroimmunol. 2005;169(1-2):31-8. https://doi.org/10.1016/j.jneuroim.2005.07.014

53. Sioofy-Khojine AB, Lehtonen J, Nurminen N, Laitinen $\mathrm{OH}$, Oikarinen S, Huhtala $\mathrm{H}$, et al. Coxsackievirus B1 infections are associated with the initiation of insulindriven autoimmunity that progresses to type 1 diabetes. Diabetologia. 2018;61(5):1193-1202. https://doi.org/10.1007/s00125-018-4561-y

54. Takahashi Y, Murai C, Shibata S, Munakata Y, Ishii T, Ishii $\mathrm{K}$, et al. Human parvovirus B19 as a causative agent for rheumatoid arthritis. Proc Natl Acad Sci U S A. 1998;95(14):8227-32. https://doi.org/10.1073/pnas.95.14.8227

55. Román GC, Gracia F, Torres A, Palacios A, Gracia K, Harris D. Acute Transverse Myelitis (ATM):Clinical Review of 43 Patients With COVID-19-Associated ATM and 3 Post-Vaccination ATM Serious Adverse Events With the ChAdOx1 nCoV-19 Vaccine (AZD1222). Front Immunol. 2021;12:653786. https://doi.org/10.3389/fimmu.2021.653786

56. Greinacher A, Thiele T, Warkentin TE, Weisser K, Kyrle PA, Eichinger S. Thrombotic Thrombocytopenia after ChAdOx1 nCov-19 Vaccination. N Engl J Med. 2021:NEJMoa2104840. https://doi.org/10.1056/NEJMoa2104840
57. Isaacs A, Cox RA, Rotem Z. Foreign nucleic acids as the stimulus to make interferon. Lancet. 1963;2(7299):113-6. https://doi.org/10.1016/s0140-6736(63)92585-6

58. Karikó K, Muramatsu H, Welsh FA, Ludwig J, Kato H, Akira $S$, et al. Incorporation of pseudouridine into mRNA yields superior nonimmunogenic vector with increased translational capacity and biological stability. Mol Ther. 2008;16(11):1833-40. https://doi.org/10.1038/mt.2008.200

59. Karikó K, Muramatsu H, Ludwig J, Weissman D. Generating the optimal mRNA for therapy: HPLC purification eliminates immune activation and improves translation of nucleoside-modified, protein-encoding mRNA. Nucleic Acids Res. 2011;39(21):e142. https://doi.org/10.1093/nar/gkr695

60. Saavedra Trujillo $\mathrm{CH}$. Consenso colombiano de atención, diagnóstico y manejo de la infección por SARS-COV-2/ COVID-19 en establecimientos de atención de la salud. Recomendaciones basadas en consenso de expertos e informadas en la evidencia. Infectio. 2020;24(3). https://doi.org/10.22354/in.v24i3.895

61. Velikova T, Georgiev T. SARS-CoV-2 vaccines and autoimmune diseases amidst the COVID-19 crisis. Rheumatol Int. 2021;41(3):509-518. https://doi.org/10.1007/s00296-021-04792-9

62. Tse F, Moayyedi P, Waschke KA, MacMillan M, Forbes N, Carroll MW, Carman N, Leontiadis GI. COVID19 Vaccination in Patients With Inflammatory Bowel Disease: Communiqué From the Canadian Association of Gastroenterology. J Can Assoc Gastroenterol. 2021;4(1):49. https://doi.org/10.1093/jcag/gwaa046

63. Melmed GY, Rubin DT, McGovern DPB. Winter Is Coming! Clinical, Immunologic, and Practical Considerations for Vaccinating Patients With Inflammatory Bowel Disease During the Coronavirus Disease-2019 Pandemic. Gastroenterology. 2021;160(3):639-644. https://doi.org/10.1053/j.gastro.2020.10.013 